\title{
Multistep Electrode Processes in Double Potential Step Techniques
}

\author{
Angela Molina*, Manuela López-Tenés, Marién M. Moreno, Carmen Serna \\ Departamento de Química Física, Facultad de Química, Universidad de Murcia, Espinardo, \\ Murcia 30100, Spain.
}

Received 15 January 2003; accepted in revised form 17 November 2003

\begin{abstract}
A rigorous analytical solution for linear diffusion corresponding to the double potential step problem for a reversible multistep process is derived. The expressions obtained are valid for any value of the formal potentials of each step and can be applied to any double pulse technique without restriction on the duration of both pulses. Differential pulse techniques are applied to the study of reversible processes with different number of steps.
\end{abstract}

Keywords: reversible multistep electrode processes, double potential step techniques, planar electrodes.

\section{Introduction}

The charge transfer processes that occur in several steps have been a subject of great interest in recent years, and have been studied in different scientific fields. Hence, for example, the well known fullerenes like $\mathrm{C}_{60}$, have shown that their reduction occurs through six successive reversible steps of one electron [1]. Nevertheless, the rigorous treatment of multistep processes with potentiostatic techniques is not simple, and in fact, has hardly been performed [2], even in the simplest case in which it is considered that only two steps occur reversibly (EE mechanism) and only then by applying, usually, dc or normal pulse techniques [3] or also numerical solutions with multipulse potential techniques $[4,5]$. We

\footnotetext{
*Corresponding author. E-mail address: amolina@um.es
} 
have recently obtained, for a reversible EE process, the solution corresponding to the application of a double potential pulse $[6,7]$ to planar electrodes, and for multistep mechanisms we have derived the general theory in voltammetry with constant potential for planar and spherical electrodes $[8,9]$.

In this paper we derive the rigorous analytical solution for a reversible multistep process in any double potential step experiment at planar electrodes. These expressions are applicable without restrictions on the duration of the two potential pulses and whatever the species initially present in the solution.

In this work we apply this solution to the study of electrochemical processes with different numbers of steps in differential double pulse techniques, such as differential normal pulse voltammetry (DNPV) and differential pulse voltammetry (DPV), the analytical expressions of which have not yet been established. These have proved, on the one hand, to be excellent and versatile techniques for trace analysis, since in contrast to other voltammetric methods, the potential time sequence can be chosen to minimise the contribution of capacitative effects in the measured current [10] and, on the other, their usefulness in detecting and characterising these processes.

\section{Theory}

Let us consider a multistep mechanism, in which $k$ reversible charge transfer reactions take place according to the following scheme,

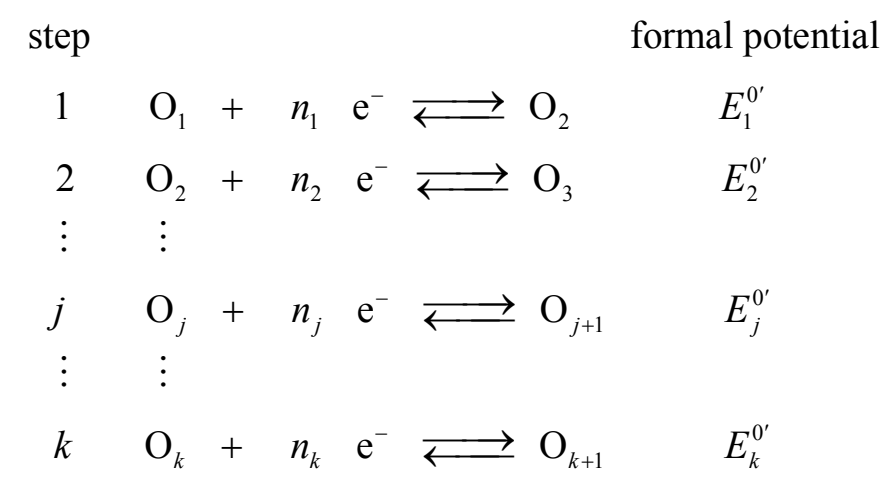

where $E_{j}^{0^{\prime}}$ and $n_{j}$ are, respectively, the formal potential and the number of electrons implied in the step $j$. We will suppose that the diffusion coefficients of the $k+1$ species participating in this process are equal $(=D)$, so the possible 
$(k-1)$ homogeneous reproportionation reactions (II) have no influence on the $I / E$ curves for any single or multipotential step voltammetric technique when linear, spherical or cylindrical semi-infinite diffusion are considered [11].

$$
n_{i+1} O_{i}+n_{i} O_{i+2 \longleftarrow}^{\stackrel{k_{i}}{\longleftrightarrow}}\left(n_{i}+n_{i+1}\right) O_{i+1} \quad i=\{1,2, \ldots, k-1\}
$$

In previous papers $[8,9]$, we have solved the problem for process (I) when a first potential pulse $E^{\mathrm{I}}$ is applied during $t_{1}$ to a planar electrode, and the analytical expressions for the profile concentrations and current have been deduced. In this paper, we will use these solutions to derive those corresponding to the application of a second potential step, $E^{\mathrm{II}}$, to a planar electrode during time $t_{2}$ following $t_{1}$. In order to do this, we present below a short outline of the problem when first potential $E^{\mathrm{I}}$ is applied.

In all expressions appearing in this work, the superindexes (I or II) refer to the number of pulse potential applied ( $E^{\mathrm{I}}$ or $E^{\mathrm{II}}$ ), whereas the subindexes refer to the species considered $(\{1,2, \ldots k+1\})$ or to the step considered $(\{1,2, \ldots k\})$ in the $k$-step process, as will be indicated in each case.

\section{First pulse}

If we consider a planar electrode when a first potential step $E=E^{\mathrm{I}}$ is applied during $t$ with $0 \leq t \leq t_{1}$, then the equation system that describes the mass transport to and from the electrode is given by

$$
\hat{\delta} c_{i}^{\mathrm{I}}(x, t)=0 \quad i=\{1,2, \ldots, k+1\}
$$

with

$$
\hat{\delta}=\frac{\partial}{\partial t}-D \frac{\partial^{2}}{\partial x^{2}}
$$

The boundary value problem is given by

$$
\left.\begin{array}{l}
t=0, x \geq 0 \\
t>0, x \rightarrow \infty
\end{array}\right\} \quad c_{i}^{\mathrm{I}}=c_{i}^{*} \quad i=\{1,2, \ldots, k+1\}
$$




$$
\begin{array}{cc}
t>0, x=0: & \sum_{i=1}^{k+1}\left(\frac{\partial c_{i}^{\mathrm{I}}}{\partial x}\right)_{x=0}=0 \\
c_{i}^{\mathrm{I}}(x=0, t)=J_{i}^{\mathrm{I}} c_{i+1}^{\mathrm{I}}(x=0, t) & i=\{1,2, \ldots, k\}
\end{array}
$$

with $J_{i}^{\mathrm{I}}$ given by

$$
J_{i}^{\mathrm{I}}=\exp \left(\frac{n_{i} F\left(E^{\mathrm{I}}-E_{i}^{0^{\prime}}\right)}{R T}\right)
$$

$c_{i}^{\mathrm{I}}(x, t)$ is the concentration of species $\mathrm{O}_{i}$ as a function of the distance from the surface electrode, $x$, and the time, $t$, and $c_{i}^{*}$ is the bulk concentration of $\mathrm{O}_{i}$ species.

This problem has an easy solution, which is given by eq. (8) in reference [9]. From this reference we can write

$$
c_{i}^{\mathrm{I}}(x, t)=c_{i}^{*}+\left[c_{i}^{\mathrm{I}}(x=0)-c_{i}^{*}\right] \operatorname{erfc}\left(\frac{x}{2 \sqrt{D t}}\right) \quad i=\{1,2, \ldots, k+1\}
$$

where

$$
\left.\begin{array}{l}
c_{i}^{\mathrm{I}}(x=0)=\frac{c^{*} \prod_{f=i}^{k} J_{f}^{\mathrm{I}}}{1+\sum_{j=1}^{k}\left(\prod_{f=j}^{k} J_{f}^{I}\right)} ; \quad i=\{1,2, \ldots k\} \\
c_{k+1}^{\mathrm{I}}(x=0)=\frac{c^{*}}{1+\sum_{j=1}^{k}\left(\prod_{f=j}^{k} J_{f}^{\mathrm{I}}\right)}
\end{array}\right\}
$$

being

$$
c^{*}=\sum_{i=1}^{k+1} c_{i}^{*}
$$

The current observed for the $k$-step mechanism, $I^{\mathrm{I}}$, is obtained by the sum 


$$
I^{\mathrm{I}}=\sum_{j=1}^{k} I_{j}^{\mathrm{I}}
$$

where $I_{j}^{\mathrm{I}}$ is the partial current due to any step $j$ and it is calculated using the expression $[8,9]$

$$
I_{j}^{\mathrm{I}}(t)=n_{j} \mathrm{~F} A D \sum_{i=1}^{j}\left(\frac{\partial c_{i}^{\mathrm{I}}}{\partial x}\right)_{x=0}
$$

By introducing eq. (7) in eq. (11) we obtain

$$
I_{j}^{\mathrm{I}}(t)=n_{j} \mathrm{~F} A\left(\frac{D}{\pi t}\right)^{1 / 2} \sum_{i=1}^{j}\left[c_{i}^{*}-c_{i}^{\mathrm{I}}(x=0)\right]
$$

and finally, the observed current is obtained with eq. (12) in eq. (10) giving

$$
I^{\mathrm{I}}(t)=\mathrm{F} A\left(\frac{D}{\pi t}\right)^{1 / 2} \sum_{i=1}^{k}\left(\sum_{j=i}^{k} n_{j}\right)\left[c_{i}^{*}-c_{i}^{\mathrm{I}}(x=0)\right]
$$

where $F$ and $A$ have their usual meanings.

\section{Second pulse}

In this situation, the equation system to solve is given by

$$
\hat{\delta} c_{i}^{\mathrm{II}}(x, t)=0 \quad i=\{1,2, \ldots, k+1\}
$$

where the total time is now $t=t_{1}+t_{2}$, with $t_{1}$ constant. The boundary value problem can be written as

$$
\begin{array}{lr}
\left.\begin{array}{l}
t_{2}=0, \\
t_{2}>0, x \rightarrow \infty
\end{array}\right\} \quad \quad c_{i}^{\mathrm{II}}=c_{i}^{\mathrm{I}}(x, t) & i=\{1,2, \ldots, k+1\} \\
t_{2}>0, x=0: & \sum_{i=1}^{k+1}\left(\frac{\partial c_{i}^{\mathrm{II}}}{\partial x}\right)_{x=0}=0
\end{array}
$$




$$
c_{i}^{\mathrm{II}}(x=0, t)=J_{i}^{\mathrm{II}} c_{i+1}^{\mathrm{II}}(x=0, t) \quad i=\{1,2, \ldots, k\}
$$

where $J_{i}^{\mathrm{II}}(i=\{1,2, \ldots, k\})$ is a function of the applied potential $E^{\mathrm{II}}$, which is given by

$$
J_{i}^{\mathrm{II}}=\exp \left(\frac{n_{i} F\left(E^{\mathrm{II}}-E_{i}^{0^{\prime}}\right)}{R T}\right)
$$

Taking into account that the operator $\hat{\delta}$ given by eq. (2) is linear, the solutions corresponding to the second potential step, $c_{i}^{\mathrm{II}}(x, t)$ can be written as (see eq. (14))

$$
c_{i}^{\mathrm{II}}(x, t)=c_{i}^{\mathrm{I}}(x, t)+\tilde{c}_{i}^{\mathrm{II}}\left(x, t_{2}\right) \quad i=\{1,2, \ldots, k+1\}
$$

where $c_{i}^{\mathrm{I}}(x, t)$ are the solutions corresponding to the first potential step $E^{\mathrm{I}}$ (see eq. (7)) and $\tilde{c}_{i}^{\mathrm{II}}\left(x, t_{2}\right)$ are the new unknown partial solutions.

By introducing eq. (19) in eqs. (14)-(17) and taking into account eqs. (1)-(5) for the first pulse, we deduce that the boundary value problem of this second pulse is given only in terms of the new unknown variables $\tilde{c}_{i}^{\text {II }}\left(x, t_{2}\right)$, and has the advantage of having null initial conditions. In effect, we have

$$
\begin{gathered}
\hat{\delta} \tilde{c}_{i}^{\mathrm{II}}\left(x, t_{2}\right)=0 \quad i=\{1,2, \ldots, k+1\} \\
\left.\begin{array}{c}
t_{2}=0, x \geq 0 \\
t_{2}>0, x \rightarrow \infty
\end{array}\right\} \quad \begin{array}{c}
\tilde{c}_{i}^{\mathrm{II}}=0 \quad\{1,2, \ldots, k+1\} \quad t_{2}>0, x=0 \\
\sum_{i=1}^{k+1}\left(\frac{\partial \tilde{c}_{i}^{\mathrm{II}}}{\partial x}\right)_{x=0}=0 \\
\tilde{c}_{i}^{\mathrm{II}}(x=0, t)=J_{i}^{\mathrm{II}} \tilde{c}_{i+1}^{\mathrm{II}}(x=0, t)+\left(J_{i}^{\mathrm{II}}-J_{i}^{\mathrm{I}}\right) c_{i+1}^{\mathrm{I}}(x=0)
\end{array} \quad i=\{1,2, \ldots, k\}
\end{gathered}
$$


Note that the conditions which must fulfil $\tilde{c}_{i}^{\mathrm{II}}(x=0)$, (eqs. (22), (23) and (18)), have a similar form to that corresponding to the first potential step, $E^{\mathrm{I}}$ (eqs. (4), (5) and (6), respectively), except in the value of the constants $\left(J_{i}^{\mathrm{II}}-J_{i}^{\mathrm{I}}\right) c_{i+1}^{\mathrm{I}}(x=0)$ in eq. (23), since the surface concentrations corresponding to the first potential step, $c_{i}^{\mathrm{I}}(x=0)$, are independent on time (see eq. (8)). Therefore, the partial solutions for the second pulse, $\tilde{c}_{i}^{\mathrm{II}}\left(r, t_{2}\right)$, have an analogous form to that corresponding to the first one (eq. (7)), with surface values also independent of time. Taking into account eqs. (19)-(23), these partial solutions are given by

$$
\tilde{c}_{i}^{\mathrm{II}}\left(x, t_{2}\right)=\left[c_{i}^{\mathrm{II}}(x=0)-c_{i}^{\mathrm{I}}(x=0)\right] \operatorname{erf} c\left[\frac{x}{2 \sqrt{D t_{2}}}\right] \quad i=\{1,2, \ldots, k+1\}
$$

with $c_{i}^{\mathrm{I}}(x=0)$ given by eq. (8) and $c_{i}^{\mathrm{II}}(x=0)$ by

$$
\begin{gathered}
c_{i}^{\mathrm{II}}(x=0)=\frac{c^{*} \prod_{f=i}^{k} J_{f}^{\mathrm{II}}}{1+\sum_{j=1}^{k}\left(\prod_{f=j}^{k} J_{f}^{\mathrm{II}}\right)} ; \quad i=1,2, \ldots k \\
c_{k+1}^{\mathrm{II}}(x=0)=\frac{c^{*}}{1+\sum_{j=1}^{k}\left(\prod_{f=j}^{k} J_{f}^{\mathrm{II}}\right)}
\end{gathered}
$$

Note that, as in the case of a reversible E simple process [12], the surface concentrations of species $\mathrm{O}_{i}$ are independent of time and take the same form for the first and second potential steps (compare eqs.(8) and (25)). Finally, by substituting eqs. (24) and (7) in eq. (19) the concentration profiles for this second pulse potential are totally determined.

The partial current due to any step $j$ (with $j=1$ to $k$ ) of the $k$-step process when a second potential step is applied is given by

$$
I_{j}^{\mathrm{II}}(t)=\mathrm{F} A D \sum_{i=1}^{j}\left(\frac{\partial c_{i}^{\mathrm{II}}}{\partial x}\right)_{x=0}
$$


and can be expressed, taking into account eqs. (19), (24) and (11), in the following way

$$
I_{j}^{\mathrm{II}}(t)=I_{j}^{\mathrm{I}}(t)+n_{j} \mathrm{~F} A D \sum_{i=1}^{j}\left[c_{i}^{\mathrm{II}}(x=0)-c_{i}^{\mathrm{I}}(x=0)\right]_{x=0} \quad j=\{1,2, \ldots, k\}
$$

with $I_{j}^{\mathrm{I}}(t)$ given by eq. (12) with $t=t_{1}+t_{2}$.

From eqs. (27) and (12) or (13), the following expression for the current observed, $I^{\mathrm{II}}(t)$, during the second potential step is obtained,

$$
\begin{aligned}
I^{\mathrm{II}}(t)=\sum_{j=1}^{k} I_{j}^{\mathrm{II}}(t)=F A\left(\frac{D}{\pi}\right)^{1 / 2} & \left\{\frac{1}{t^{1 / 2}} \sum_{i=1}^{k}\left(\sum_{j=i}^{k} n_{j}\right)\left[c_{i}^{*}-c_{i}^{\mathrm{I}}(x=0)\right]+\right. \\
& \left.+\frac{1}{t_{2}^{1 / 2}} \sum_{i=1}^{k}\left(\sum_{j=i}^{k} n_{j}\right)\left[c_{i}^{\mathrm{I}}(x=0)-c_{i}^{\mathrm{II}}(x=0)\right]\right\}
\end{aligned}
$$

which is given by a sum of two terms corresponding to two single potential steps. This equation is the general current in planar diffusion for reversible multistep electrode processes with any number of steps $(k)$ and whatever the values of the formal potentials $E_{1}^{0^{\prime}}, E_{2}^{0^{\prime}}, \ldots, E_{k}^{0^{\prime}}$ (i. e., whatever the species, $\mathrm{O}_{i}(i=1,2, \ldots k+1)$ initially present in the solution), and can be used without restriction on the duration of both pulses.

The expressions for the currents corresponding to the first and second potential pulse (eqs. (13) and (28), respectively), can be written as

$$
\begin{gathered}
I^{\mathrm{I}}=\frac{F A c^{*} \sqrt{D}}{\sqrt{\pi t_{1}}}\left(X^{\mathrm{I}}-X^{\phi}\right) \\
I^{\mathrm{II}}=\frac{F A c^{*} \sqrt{D}}{\sqrt{\pi t_{2}}}\left\{\sqrt{\frac{t_{2}}{t_{1}+t_{2}}}\left(X^{\mathrm{I}}-X^{\phi}\right)+\left(X^{\mathrm{II}}-X^{\mathrm{I}}\right)\right\}
\end{gathered}
$$

where $X^{\phi}, X^{\mathrm{I}}$ and $X^{\mathrm{II}}$ are given by

$$
X^{\phi}=\frac{1}{c^{*}}\left\{n_{k} c_{k+1}^{*}-\sum_{i=1}^{k-1}\left(\sum_{j=i}^{k-1} n_{j}\right) c_{i}^{*}\right\}
$$




$$
\begin{aligned}
& X^{\mathrm{I}}=\frac{1}{1+\sum_{m=1}^{k} \prod_{f=m}^{k} J_{f}^{\mathrm{I}}}\left\{n_{k}-\sum_{i=1}^{k-1}\left(\sum_{j=i}^{k-1} n_{j}\right) \prod_{f=i}^{k} J_{f}^{\mathrm{I}}\right\} \\
& X^{\mathrm{II}}=\frac{1}{1+\sum_{m=1}^{k} \prod_{f=m}^{k} J_{f}^{\mathrm{II}}}\left\{n_{k}-\sum_{i=1}^{k-1}\left(\sum_{j=i}^{k-1} n_{j}\right) \prod_{f=i}^{k} J_{f}^{\mathrm{II}}\right\}
\end{aligned}
$$

When $k=2$, all the above equations obtained for the second potential step coincide with those deduced in Ref. [7] for an EE mechanism when planar electrodes are used.

\section{Differential pulse techniques}

\section{a. Differential normal pulse voltammetry (DNPV)}

In the double pulse technique DNPV two consecutive potential steps, $E^{\mathrm{I}}$ and $E^{\mathrm{II}}$, are applied during times $t_{1}$ and $t_{2}$, respectively, without restriction on the duration of either pulse, and the difference $\Delta E=E^{\mathrm{II}}-E^{\mathrm{I}}$ is kept constant during the experiment. Currents $I^{\mathrm{I}}$ and $I^{\mathrm{II}}$ are measured at the end of each pulse and the difference $\Delta I_{\mathrm{DNPV}}=I^{\mathrm{II}}-I^{\mathrm{I}}$ is plotted versus $E^{\mathrm{I}}$. Thus, by subtracting eq. (30) and eq. (29) we can obtain the expression for the response of a reversible multistep process in DNPV, which is given by

$$
\psi_{D N P V}=\left(\left(\frac{t_{2}}{t_{1}+t_{2}}\right)^{1 / 2}-\left(\frac{t_{2}}{t_{1}}\right)^{1 / 2}\right)\left(X^{\mathrm{I}}-X^{\phi}\right)+\left(X^{\mathrm{II}}-X^{\mathrm{I}}\right)
$$

where

$$
\psi_{\mathrm{DNPV}}=\frac{\Delta I_{\mathrm{DNPV}}}{F A c^{*}\left(D / \pi t_{2}\right)^{1 / 2}}
$$


The expressions for this response in the cathodic and anodic limits can be deduced from eq. (34) by making $E^{\mathrm{I}}, E^{\mathrm{II}} \rightarrow-\infty$ and $E^{\mathrm{I}}, E^{\mathrm{II}} \rightarrow \infty$, respectively in eqs. (32), (33), (6) and (18). Thus, we obtain

$$
\begin{gathered}
\psi_{\mathrm{DNPV}}^{\mathrm{c} .1 \mathrm{~g}}=\left(\left(\frac{t_{2}}{t_{1}+t_{2}}\right)^{1 / 2}-\left(\frac{t_{2}}{t_{1}}\right)^{1 / 2}\right) \frac{\sum_{i=1}^{k}\left(\sum_{j=i}^{k} n_{j}\right) c_{i}^{*}}{c^{*}} \\
\psi_{\mathrm{DNPV}}^{\text {a.l. }}=-\left(\left(\frac{t_{2}}{t_{1}+t_{2}}\right)^{1 / 2}-\left(\frac{t_{2}}{t_{1}}\right)^{1 / 2}\right) \frac{\sum_{i=2}^{k+1}\left(\sum_{j=i}^{k} n_{j}\right) c_{i}^{*}}{c^{*}}
\end{gathered}
$$

Note that these limit currents are dependent on the initial concentrations of the species participating in process (I).

\section{b. Differential pulse voltammetry (DPV)}

If we impose the condition $t_{2}<<t_{1}$, the first term in eq. (34) can be suppressed and the expression for the current corresponding to DPV is obtained

$$
\psi_{\mathrm{DPV}}=\left(X^{\mathrm{II}}-X^{\mathrm{I}}\right)
$$

Note that both limit currents in the DPV are always null (see eqs. (34) and (35) with $\left.t_{2}<<t_{1}\right)$ and also that the signal in this technique is independent of which species are initially present in the solution (see eq.(38)).

Eq. (38) is applicable to any $k$-step reversible process without restriction on the values of the formal potential of each step. The multistep mechanisms for which the values of the formal potentials verify that $E_{1}^{0^{\prime}}>>\ldots E_{s}^{0^{\prime}}>>\ldots>E_{k}^{0^{\prime}}$ always present well-separated $k$ responses. In this case, for the response corresponding to any step $s$ it is verified that 


$$
\begin{aligned}
& J_{j<s}^{\mathrm{p}}=0 \\
& J_{s}^{\mathrm{p}}=\exp \left(\frac{n_{s} F\left(E^{\mathrm{p}}-E_{s}^{0^{\prime}}\right)}{R T}\right) \quad \text { with } \mathrm{p}=\mathrm{I}, \mathrm{II} \\
& J_{j>s}^{\mathrm{p}} \rightarrow \infty
\end{aligned}
$$

By introducing the above expressions in eq. (38) for the double pulse techniques DPV, we obtain the response corresponding to any step, whose expression is given by

$$
\left(\psi_{\mathrm{DPV}}\right)_{s}=\left(\frac{1}{1+J_{s}^{\mathrm{II}}}-\frac{1}{1+J_{s}^{\mathrm{I}}}\right)
$$

This equation for the normalised curve is coincident with those corresponding to a simple E mechanism [13] and therefore, it is possible to deduce the expressions for the formal potentials of each step, obtaining

$$
E_{s}^{0^{\prime}}=E_{\text {peak }} \mp \frac{|\Delta E|}{2}
$$

where the upper and lower signs in eq. (41) refer to the cathodic and anodic peaks obtained in DPV curves, when the pulse amplitude, $\Delta E$, is applied with a negative or positive sign, respectively.

\section{Results and discussion}

Fig. 1 shows the influence of the duration of the second potential step, $t_{2}$, on the normalized DNP curves corresponding to a reversible EEE process (eq.(34) with $k=3)$ by supposing that $n_{1}=n_{2}=n_{3}=1$ and $E_{z+1}^{0^{\prime}}-E_{z}^{0^{\prime}}=-200 \mathrm{mV}(z=1,2)$ for $\Delta E=-50 \mathrm{mV}$ when species $O_{1}$ and $O_{4}$ are initially present in the solution with concentrations $c_{1}^{*}=c_{4}^{*}=1 \mathrm{mM}$. As can be observed in this figure, when the electrode processes have several completely separated steps $\left(E_{z+1}^{0^{\prime}}-E_{z}^{0^{\prime}} \leq-200 \mathrm{mV}\right)$, the $\psi_{\mathrm{DNPV}}$ or $\psi_{D P V}$ curves obtained always present a well defined peak for each step, with a height that increases in absolute value when $t_{2}$ 
decreases. Hence the analytical sensitivity of the technique is greater for lower values of $t_{2}$. Fig. 1 also shows how the values of both limit currents tend to zero when $t_{2}$ decreases, and so the characteristic response in DPV is reached for $t_{2} \ll<t_{1}$ (see curve with $t_{2}=0.02$ in Fig. 1). Note that the response in DPV is independent of which species are initially present in the solution but always depends on the sum of all the initial concentrations.

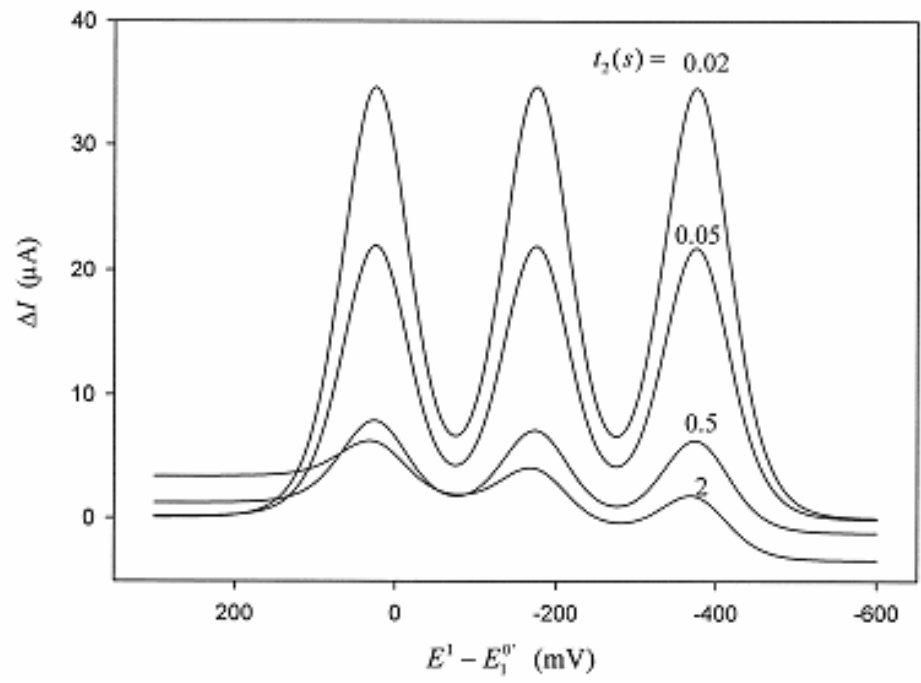

Figure 1. Influence of the duration of the second potential pulse, $t_{2}$, on the differential normal pulse voltammograms, $\Delta I_{\mathrm{DNPV}} /\left(E^{\mathrm{I}}-E_{1}^{0^{\prime}}\right)$ for a reversible EEE with completely separated steps, $E_{2}^{0^{\prime}}-E_{1}^{0^{\prime}}=E_{3}^{0^{\prime}}-E_{2}^{0^{\prime}}=-200 \mathrm{mV}$, when $\mathrm{O}_{1}$ and $\mathrm{O}_{4}$ are initially present in the solution (eqs. (34) and (35) with $k=3$ ) with concentrations $c_{1}^{*}=c_{4}^{*}=1 \mathrm{mM}$, $T=298 \mathrm{~K}, n_{1}=n_{2}=n_{3}=1, D=10^{-5} \mathrm{~cm}^{2} \mathrm{~s}^{-1}, \Delta E=-50 \mathrm{mV}, t_{1}=2 \mathrm{~s}$. The values of $t_{2}$ (in s) are on the curves.

In Fig. 2 we have represented the $\psi_{\mathrm{DPV}} /\left(E^{\mathrm{I}}-E_{1}^{0^{\prime}}\right)$ curves corresponding to a reversible EEE process with $n_{1}=n_{2}=n_{3}=1$ and $\Delta E=-50 \mathrm{mV}$ at different values of $E_{z+1}^{0^{\prime}}-E_{z}^{0^{\prime}}(z=1,2)$. From this figure we deduce that the height of a peak for an EEE mechanism with $E_{z+1}^{0^{\prime}}-E_{z}^{0^{\prime}} \leq 100 \mathrm{mV}$ is considerably lower than that corresponding to a simple E process with three electrons (which is coincident with the curves of $E_{z+1}^{0^{\prime}}-E_{z}^{0^{\prime}} \geq 150 \mathrm{mV}$ in this figure), with a relative difference in heights of more than 5\%. Therefore, both processes can be perfectly distinguishable in DPV even when the EEE mechanism only presents one peak 
(see curves corresponding to 50, 0 and $-50 \mathrm{mV}$ ). From the above we can conclude that, when the formal potentials of both steps are very close, DPV technique is more useful for detecting a three step process than other techniques of single or double pulse, like Normal (NP) and Reverse Pulse (RP) Voltammetries, since in the latter, the limit currents remain independent of the value of $E_{z+1}^{0^{\prime}}-E_{z}^{0^{\prime}}$ and, for example, for $E_{z+1}^{0^{\prime}}-E_{z}^{0^{\prime}} \cong 0$ the shape-wave in NP or RP curves is only slightly different from that corresponding to an E simple process with three electrons (see Fig. 3b).

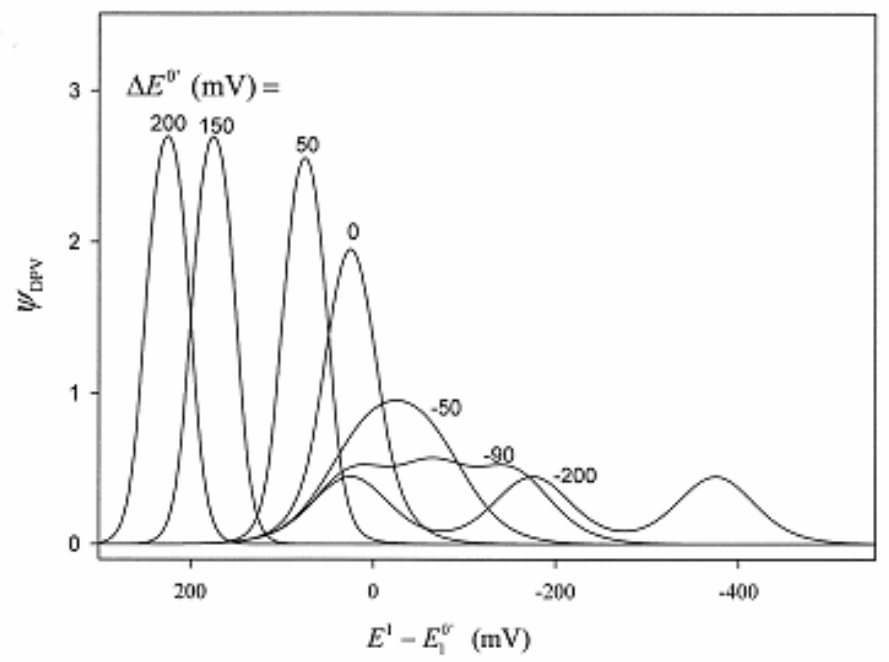

Figure 2. Influence of the value of the separation between the formal potentials of consecutive steps, $\Delta E_{j}^{0^{\prime}}=E_{j+1}^{0^{\prime}}-E_{j}^{0^{\prime}} \quad(j=1,2)$, on the differential pulse voltammograms, $\psi_{\mathrm{DPV}} /\left(E^{\mathrm{I}}-E_{1}^{0^{\prime}}\right)$, for an EEE mechanism (eq. (38) with $k=3$ ) when only $\mathrm{O}_{1}$ is initially present in the solution with concentration $c_{1}^{*}=1 \mathrm{mM} . \Delta E_{1}^{0^{\prime}}=\Delta E_{2}^{0^{\prime}}$, $t_{1}=2 \mathrm{~s}, t_{2}=0.01 \mathrm{~s}$. The values of $\Delta E_{j}^{0^{\prime}}$ (in $\mathrm{mV}$ ) are on the curves. Other conditions as in Fig. 1.

Fig. 3a shows the influence of pulse amplitude, $\Delta E$, on $\psi_{\mathrm{DPV}} /\left(E^{\mathrm{I}}-E_{1}^{0^{0}}\right)$ curves. From this figure it is clear that for a given value of $\Delta E^{0^{\prime}}(-80 \mathrm{mV}$ in this case), a decrease of $|\Delta E|$ tends to decrease the current of the $\psi_{\mathrm{DPV}}$ curves and tends to separate out into four peaks. Thus, by acting on the experimental conditions it is possible to detect at sight the existence of a multistep process with DPV, whereas 
it would be impossible to carry out with dc voltammetry, as can be seen in Fig. $3 \mathrm{~b}$, which shows the current $I^{\mathrm{I}} /\left(E^{\mathrm{I}}-E_{1}^{0^{\prime}}\right)$ for the same process.

Figure 3a

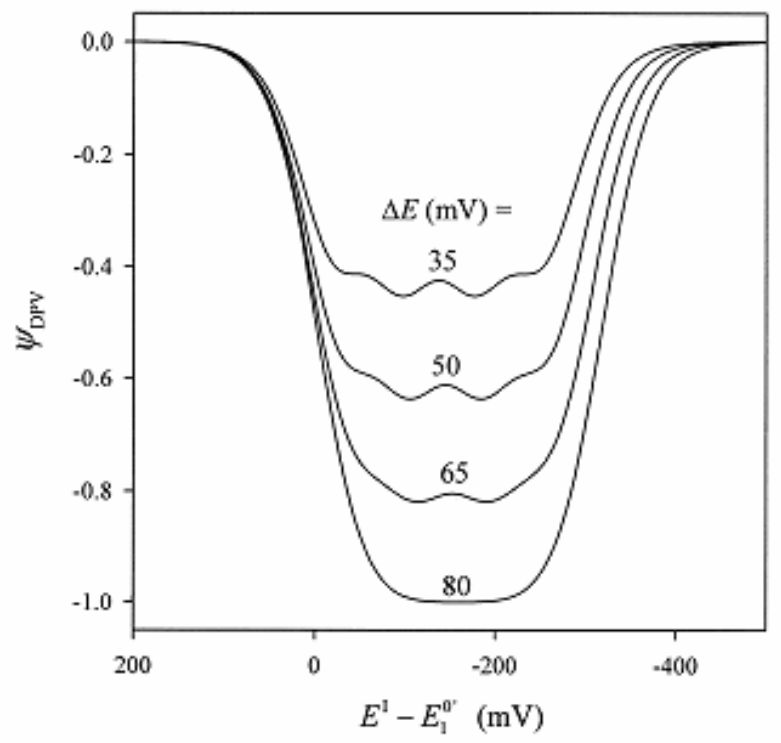

Figure 3b

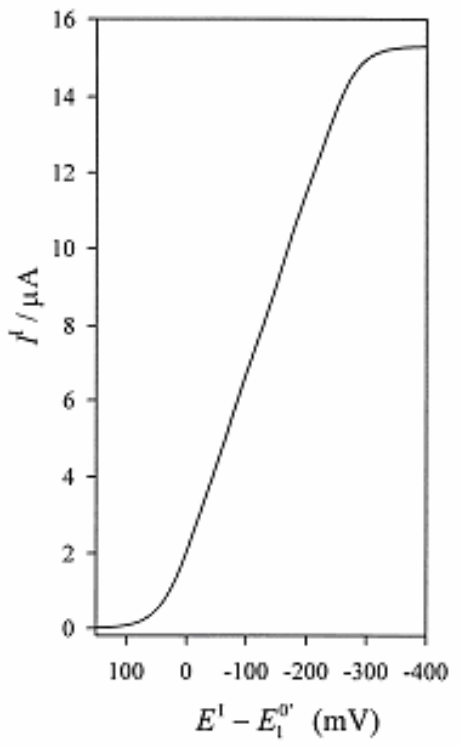

Figure 3. Influence of pulse amplitude, $\Delta E$, on the differential pulse voltammograms $\Delta I_{\mathrm{DPV}} /\left(E^{\mathrm{I}}-E_{1}^{0^{\prime}}\right)$, for a reversible four-step mechanism (eq. (38) with $\left.k=4\right)$. $E_{z+1}^{0^{\prime}}-E_{z}^{0^{\prime}}=-80 \mathrm{mV}(z=1,2,3), n_{j}=1 \quad(j=1,2,3,4)$. The values of $\Delta E$ (in $\mathrm{mV}$ ) are on the curves. Other conditions as in Fig. 2.

\section{Acknowledgements}

The authors greatly appreciate the financial support provided by the Dirección General Científica y Técnica (Project No. BQU2000-0231), and by the Fundación Séneca (Projects 00696/CV/99 and AR 28-02698/FS/02). Also M. M. M. thanks Dirección General Científica y Técnica for the grant received.

\section{References}

1. G. Diao, Z. Zhang, J. Electroanal. Chem. 414 (1996) 177-181.

2. C. Amatore, S.C. Paulson, H.S. White, J. Electroanal. Chem. 493 (1997) 173182.

3. M. Rueda, M. Sluyters-Rehbach, J.H. Sluyters, J. Electroanal. Chem. 202 (1986) 271-297. 
4. K. Tsaur, R. Pollard, J. Electroanal. Chem. 183 (1985) 91-106.

5. I. Prieto, J.M. Pedrosa, M.T. Martín, L. Camacho, J. Electroanal. Chem. 485 (2000) 7-12.

6. M.M. Moreno, A. Molina, C. Serna, M. López-Tenés, Portugaliae Electrochim. Acta 19 (2001) 325-336.

7. M. López-Tenés, M.M. Moreno, C. Serna, A. Molina, J. Electroanal. Chem. 528 (2002) 159-169.

8. C. Serna, M. López-Tenés, J. González, A. Molina, Electrochim. Acta 46 (2001) 2699-2709.

9. A. Molina, C. Serna, M. López-Tenés, R. Chicón, Electrochem. Commun. 2 (2000) 267-271.

10. D.J. Myers, J. Osteryoung, Anal. Chem. 46 (1974) 356-359.

11. C. Serna, A. Molina, M.M. Moreno, M. López-Tenés, J. Electroanal. Chem. (submitted).

12. C. Serna, A. Molina, J. Electroanal. Chem. 466 (1999) 8-14.

13.D. Krulic, N. Fatouros, M.M. El Belamachi, J. Electroanal. Chem. 385 (1995) 33. 